\title{
Ethisch geleitete Netzwerkforschung in inklusiven Grundschulklassen: Ein Vergleich zweier Methoden
}

\author{
Fabian Mumenthaler (D) $\cdot$ Michael Eckhart $\cdot$ Siegfried Nagel $\mathbb{D}$
}

Eingegangen: 1. November 2020 / Angenommen: 16. Juli 2021 / Online publiziert: 18. Januar 2022 (C) Der/die Autor(en) 2022

Zusammenfassung Soziometrische Verfahren stoßen häufig auf ethische Bedenken. Sie bedürfen deshalb einer besonderen Legitimierung - insbesondere in der Inklusionsforschung. In dieser Studie wird argumentiert, dass der empirische Nachweis ethisch vertretbarer Instrumente schwierig zu liefern ist, weshalb ethische Aspekte bereits in der Testkonstruktion beachtet werden müssen. Mittels Intrarater-Reliabilität bei 109 und exponential random graph models bei 77 inklusiven Grundschulklassen wird überprüft, ob zwei soziometrische Erhebungsmethoden - eine gängige Freundschaftsnomination und ein ethisch durchdachtes Interaktionsrating (Sozio) zu ähnlichen Ergebnissen führen. Der Vergleich zeigt, dass sich Freundschafts- und Interaktionsnetzwerke stark gleichen.

Schlüsselwörter Inklusive Bildung · Soziale Netzwerke · Kognitive Beeinträchtigung $\cdot$ ERGM $\cdot$ Peernomination

Fabian Mumenthaler $(\bowtie) \cdot$ Prof. Dr. Michael Eckhart Institut für Heilpädagogik, PHBern, Fabrikstrasse 8, 3012 Bern, Schweiz

E-Mail: fabian.mumenthaler@phbern.ch

Prof. Dr. Michael Eckhart

E-Mail: michael.eckhart@phbern.ch

Fabian Mumenthaler · Prof. Dr. Siegfried Nagel

Institut für Sportwissenschaft, Universität Bern, Bremgartenstrasse 145, 3012 Bern, Schweiz

E-Mail: siegfried.nagel@ispw.unibe.ch 


\title{
Ethically guided research in network science in inclusive primary classes: A comparison of two methods
}

\begin{abstract}
Sociometric procedures often face ethical concerns. Therefore, the need for legitimization is high-especially in inclusion research. In this study it is argued that it is difficult to provide empirical evidence for ethically acceptable instruments. Thus, ethical aspects should already be considered in test construction. Using intrarater reliability with 109 and exponential random graph models with 77 inclusive primary classes we test whether two sociometric survey methods lead to similar results: one is a common friendship peer nomination; the other is an ethically thoughtthrough interaction peer rating (Sozio). The comparison shows a strong similarity between the friendship and interaction networks.
\end{abstract}

Keywords Inclusive education - Social networks - Cognitive impairment - Ergm • Peer nomination

\section{Einleitung}

Die Integration von Kindern mit besonderen Bedürfnissen in die Regelschule wurde durch den Artikel 24 im Übereinkommen über die Rechte von Menschen mit Behinderungen politisch verbindlich anerkannt. Seither ist die Integration im inklusiven Schulsetting ein weltweit viel untersuchter Forschungsgegenstand (vgl. Koster et al. 2009). Die Literaturrecherche von Koster et al. (2009) über die in der Forschung verwendeten Begriffe zeigt allerdings, dass die Termini soziale Partizipation, soziale Integration und soziale Inklusion uneinheitlich verwendet und operationalisiert werden ${ }^{1}$. Ein wichtiges Konzept für die Operationalisierung ist die Soziometrie von Moreno (1996 [1974]) aus den 1930er-Jahren, welche sich zur heutigen Netzwerkanalyse weiterentwickelte. Das Nominationsverfahren von Moreno (1996), bei dem für ein zu bestimmendes Kriterium drei positive und drei negative Wahlen getroffen werden, wurde von Peery (1979) zu den Massen social impact (Summe der positiven und negativen Wahlen für ein Individuum) und social preference (Differenz aus positiven und negativen Wahlen) weiterverarbeitet, welche wiederum die Grundlage des Klassifikationsmodells von Coie et al. (1982) bilden. Coie et al.'s (1982) Klassifikationsmodell gilt häufig als Standardmodell, um den sozialen Status zu berechnen (Cillessen und Marks 2011). Auch heute wird ihr Klassifikationsmodell noch angewendet, obwohl es mindestens seit den 1970er-Jahren Autorinnen und Autoren gibt, die das Wahlverfahren - insbesondere die Negativwahl - als ethisch bedenklich einstufen (z. B. Bell-Dolan und Wessler 1994; Hayvren und Hymel 1984). Child und Nind (2012, S. 1) kritisieren die Fokussierung auf diese Status-Kategorien: „This practice of categorising individuals as popular, and so on, may construct and reinforce social difficulties, especially for disabled children“. Die moderne Netzwerkforschung ist nun von der Kategorisierung nach sozialem Status weggekommen und

\footnotetext{
1 Hier werden inklusive Klassen so verstanden, dass Kinder mit sonderpädagogischem Förderbedarf (SPF) gemeinsam mit Kindern ohne SPF in Regelklassen unterrichtet werden.
} 
verrechnet die erfassten Nennungen direkt in statistischen Modellen (Hunter et al. 2008; Snijders et al. 2010).

\subsection{Ethik und Soziometrie}

Auf ethische Bedenken bei soziometrischen Verfahren wird häufig in sonderpädagogischen Studien hingewiesen, jedoch sind sie selten der explizite Gegenstand der Forschung. Ein häufig geäußertes Bedenken ist, dass der Eingriff der Peernominierung oder des -ratings einen Einfluss auf die soziale Gruppe habe und die „risikoreichen“ Kinder noch stärker exkludiert werden könnten, als dies bereits der Fall ist (z. B. Eckhart 2012; Mayeux et al. 2007). Obwohl diese Befürchtung nicht empirisch gestützt wird, wird sie von Eltern, Lehrpersonen und Forschenden geäußert (Hayvren und Hymel 1984). Gerade bei der Forschung zur inklusiven Schule, wo es um die Erfassung der sozialen Partizipation und Integration von Kindern mit sonderpädagogischem Förderbedarf (SPF) geht, sollten solche Bedenken besondere Aufmerksamkeit erhalten.

Es können zwei verschiedene Ansätze im Umgang mit ethischen Bedenken in der Grundschulforschung festgestellt werden. Einerseits wurde empirisch untersucht, ob verschiedene soziometrische Verfahren negative Auswirkungen auf die teilnehmenden Kinder haben (z. B. Iverson und Iverson 1996) und andererseits wurde versucht, Messinstrumente konzeptuell so zu gestalten, dass sie ethisch vertretbar sind (z.B. Sozio von Eckhart 2005).

\subsection{Ziel dieser Studie}

Aufgrund ethischer Bedenken muss bei soziometrischen Instrumenten besonderer Wert auf ihre Legitimation gelegt werden. Wie im nächsten Kapitel jedoch dargelegt wird, ist diese durch empirische Untersuchungen schwierig zu liefern. Instrumente, die in ihrer Konzeption explizit ethische Aspekte bedenken, sind deshalb für die (sonderpädagogische) Forschung besser vertretbar.

In diesem Artikel wird zuerst untersucht, inwiefern das durch Eckhart (2005) weiterverarbeitete Peerrating von Krüger (1976), bei dem wahrgenommene Sprech- und Ärgerkontakte erfragt werden und das aufgrund ethischer Überlegungen entstanden ist, mit einer gängigen Freundschaftsnomination übereinstimmt. Danach wird inhaltlich analysiert, ob durch das Peerrating und die Peernomination soziale Netzwerke entstehen, welche mit den gleichen sozialen Prozessen und exogenen Faktoren erklärt werden können. Wenn die beiden Erhebungsmethoden stark übereinstimmen und zu sehr ähnlichen inhaltlichen Ergebnissen führen, so würde Sozio (Eckhart 2012) für die weitere Verwendung in der (Inklusions-)Forschung vorgeschlagen. 


\section{Ethische Diskussionen zu soziometrischen Verfahren}

\subsection{Empirische Ergebnisse}

In der neueren Inklusionsforschung besteht ein Konsens, wonach die Ergebnisse aus soziometrischen Studien dafürsprechen, dass Kinder mit SPF in Regelschulen signifikant weniger Freundschaftsnennungen und Interaktionen erhalten als ihre Mitschülerinnen und Mitschüler ohne SPF - bei gleichzeitig gleich vielen ausgehenden Freundschafts- und Interaktionsnennungen wie diese (z.B. Garrote 2016; Henke et al. 2017).

Mayeux et al. (2007) weisen auf die Paradoxie hin, dass mittels soziometrischer Verfahren die soziale Entwicklung von Kindern und ihre soziale Integration untersucht und im besten Fall verbessert werden soll, jedoch z.T. die Kinder aufgefordert werden, sich an störende oder verletzende Erfahrungen zu erinnern, wodurch sie möglicherweise eine vorher nicht wahrgenommene Zurückweisung oder Ablehnung spüren. Die bereits genannten Bedenken gegenüber der Soziometrie führten bereits so weit, dass selbst ethisch durchdachte und von Behörden unterstützte Forschungsprojekte aufgrund von elterlichen Beschwerden abgebrochen werden mussten (siehe Underwood et al. 2006, S. 95-97). Bei Krüger (1976) werden diese Bedenken so formuliert, dass je nach Kriterium und Entwicklungsstand der Kinder eine noch nicht sprachlich gefestigte oder bewusste Beurteilung über andere Kinder evoziert und gefestigt werden könne. Aus diesem Grund sollten nur Kriterien erfragt werden, welche in der Erfahrungswelt der Kinder bereits existieren - also z. B. Sprechen und Ärgern. Zudem weist Krüger (1976) darauf hin, dass mit seiner Methode die sozialen Interaktionen beurteilt werden und nicht der Mitschüler oder die Mitschülerin.

Die wenigen empirischen Untersuchungen, die zu den Auswirkungen soziometrischer Testungen vorliegen (Hayvren und Hymel 1984; Iverson und Iverson 1996; Mayeux et al. 2007), kommen allesamt zum Schluss, dass es keine Evidenz für negative Auswirkungen auf das Verhalten in der Gruppe oder auf die Gefühle der Kinder gibt. Alle drei Studien weisen jedoch methodische Mängel auf. So beruhen Hayvren und Hymel's (1984) Analyse auf lediglich zwei Schulklassen $(N=27)$ und die Überprüfung des Impacts der Erhebung erfolgte mittels zeitlich sehr limitierter Beobachtung. In der Studie von Iverson und Iverson (1996) wurde das soziometrische Verfahren in der Woche vor den Sommerferien im Mai durchgeführt und nach den Ferien im September wurden Kinder in Einzelinterviews über ihre Reaktionen zum Test befragt, wobei die Stichprobe im September systematisch verzerrt war, da vorwiegend beliebtere Kinder an der zweiten Erhebung teilnehmen durften. Bei Mayeux et al. (2007) konnten lediglich 91 Kinder aus der 3. Klasse von potenziell 382 Kindern an der soziometrischen Untersuchung und am nachfolgenden Interview teilnehmen. Das Interview zu den Reaktionen auf die soziometrische Erhebung wurde zwischen 7 und 31 Wochen nach der ersten Testung durchgeführt. Diese Zeitspanne wird auch von der Autorengruppe hinsichtlich des Erinnerungsvermögens der Kinder kritisch gesehen. Die empirische Datenlage muss als defizitär bezeichnet werden und mehr empirische Untersuchungen sind wünschenswert. Derweil besteht ein erfolgversprechender Ansatz darin, ethische Aspekte bereits in der Instrumentenkonstruktion zu berücksichtigen. 


\subsection{Prinzipien und Standards}

Bell-Dolan und Wessler (1994) und Underwood et al. (2006) verweisen für die Verwendung von soziometrischen Methoden auf die Übereinstimmung mit den ethischen Prinzipien für Psychologinnen und Psychologen und dem Verhaltenskodex (ethischer Kodex) der American Psychological Association (APA). Im ethischen Kodex werden keine Instrumente pauschal abgelehnt. Für jede neue Untersuchung sollten die Kriterien durchgegangen und reflektiert werden, ob die eingesetzten Methoden ethisch vertretbar sind. Nachfolgend werden passende Prinzipien und ethische Standards aus dem ethischen Kodex der APA (2017) auf die beiden verwendeten Methoden angewandt.

Prinzip A handelt vom Wohlwollen und der Schadensvermeidung. Da das ultimative Ziel soziometrischer Untersuchungen generell - und in der Sonderpädagogik besonders - darin liegt, die Situation von sozial exkludierten oder vernachlässigten Kindern besser zu verstehen, um sie besser zu integrieren, ist das Wohlwollen sicherlich gegeben. Bei der Schadensvermeidung sollte es darum gehen, die eingesetzten Instrumente so taktvoll (,decent“ in Underwood et al. 2006, S. 106) wie möglich zu wählen oder zu gestalten, um die sozialen Prozesse in den untersuchten Klassen nicht zu verändern (Krüger 1976). Hierzu können Unterschiede zwischen der Nomination und dem Rating festgehalten werden: Bei der Nomination werden häufig auch negative Nennungen erfragt, was auf die Moreno'sche Tradition zurückzuführen ist und auch für die klassische Kategorisierung nach Coie et al. (1982) gebraucht wird. Wie bereits ausgeführt, ist genau dies der Auslöser für die häufig vorgebrachten ethischen Bedenken. Weiter könnte argumentiert werden, dass selbst Positivwahlen immer eine implizite Negativwahl bedeuten, womit allenfalls nicht bewusste affektive Zustände verfestigt werden könnten. Beim Rating kommt es auch häufig vor, dass negative Verhaltensweisen beurteilt werden. Diese haben ihre Berechtigung, wenn sie den explizit zu untersuchenden Gegenstand darstellen (Underwood et al. 2006). Dennoch kann aus einem ethischen Gesichtspunkt bemängelt werden, dass bei diesen Ratingverfahren suggeriert wird, dass die zu beurteilenden Verhaltensweisen dem beurteilten Kind als festes Attribut zugeschrieben werden können. Das Instrument Sozio versucht diese Kritik zu vermeiden, indem klargestellt wird, dass die wahrgenommene Interaktion (Sprechen und Ärgern) zwischen zwei Kindern beurteilt wird und dass diese zu einem anderen Zeitpunkt anders aussehen kann. Dadurch werden potenziell problematische, affektive Zuschreibungen vermieden.

Prinzip B - Redlichkeit und Verantwortung - gibt vor, dass Forschende die Schulleitungen, Lehrpersonen, Eltern und Kinder ehrlich über die Forschungsinteressen und -inhalte aufklären und mögliche Bedenken ernst nehmen (Underwood et al. 2006).

Prinzip C ist Integrität. In soziometrischer Forschung kann die Integrität eingehalten werden, indem z. B. keine Daten zu Kindern ohne Einverständnis erhoben werden. Täuschungen (z. B. über Forschungsinteressen) sind ethisch vertretbar, wenn dadurch bessere Erkenntnisse gewonnen und Schaden verhindert werden kann.

Prinzip D - Gerechtigkeit - beinhaltet, dass die Untersuchungsteilnehmerinnen und -teilnehmer gleichbehandelt werden. Im sonderpädagogischen Setting kann dies bedeuten, dass alle Kinder gemeinsam befragt werden - also z. B. nicht Kinder mit 
SPF in einem abgesonderten Raum - und dass durch individuelle Hilfestellungen sichergestellt wird, dass alle die Fragen und Items verstehen und ausfüllen können.

Der ethische Standard 9 - Assessment - verlangt, dass die angewendeten Forschungsinstrumente den neuesten Standards entsprechen und obsolete und überholte Methoden nicht verwendet werden (9.08). Bei der Peernomination werden häufig auf drei oder fünf Nennungen limitierte Verfahren gewählt (vgl. Cillessen und Marks 2017). Cillessen und Marks (2017) erläutern, dass solche limitierten Verfahren nicht ökologisch valide sind. Es wurde befürchtet, dass Kinder bei gänzlicher Aufhebung der Limitierung wahllos Freundinnen und Freunde aufschreiben könnten, weshalb in unserer Studie auf neun Nennungen beschränkt wurde.

\section{Fragestellungen}

Die häufigen ethischen Bedenken weisen darauf hin, dass die soziometrischen Instrumente einer guten Legitimation bedürfen. Eine empirische Überprüfung der ethischen Vertretbarkeit scheint jedoch schwierig, weshalb ethische Aspekte bereits in der Instrumentenkonstruktion mitgedacht werden sollten. Durch die Beantwortung der folgenden Fragen soll in dieser Studie überprüft werden, ob in der Inklusionsforschung eine gewöhnliche Peernomination durch ein ethisch durchdachteres Erhebungsverfahren ersetzt werden sollte.

1. Wie stark stimmen die zwei soziometrischen Verfahren Peernomination (Freundschaft) und Sozio (Interaktion) überein?

2. Inwiefern unterscheiden sich soziale Netzwerkanalysen von Freundschaftsnennungen und Interaktionsratings inhaltlich - unter Einbezug von endogenen und exogenen Kontrollvariablen?

\section{Methodik}

Die Daten für die vorliegende Studie stammen aus dem Projekt „Soziale Partizipation im Sport“ (SoPariS), das vom Schweizerischen Nationalfonds unterstützt wird. Für das Projekt wurden insgesamt 109 inklusive Primarschulklassen (3. bis 6. Klasse) aus der Deutschschweiz befragt. Da die Gruppe der Kinder mit SPF sehr heterogen ist, wurde sie auf Kinder mit SPF aufgrund einer kognitiven Beeinträchtigung (KB) eingegrenzt. Die Kinder mit einer KB wurden von einer offiziellen Stelle abgeklärt (standardisiertes Abklärungsverfahren) und haben einen ausgewiesenen Anspruch auf verstärkte Massnahmen (vgl. EDK 2014).

\subsection{Stichprobe}

Die erste Fragestellung wird mittels Intrarater-Reliabilität (Cohen's Kappa) beantwortet, wobei die Daten von allen 109 Klassen einbezogen werden können. Die zweite Fragestellung wird mittels exponential random graph models (ERGMs) untersucht. Dabei konvergierten in der Analyse nur 77 Netzwerke. In jeder Klasse wird 
Tab. 1 Stichprobendeskription

\begin{tabular}{lllll}
\hline & \multicolumn{2}{l}{ Intrarater-Reliabilität } & \multicolumn{2}{l}{ ERGMs } \\
& Kinder mit KB & Kinder ohne KB & Kinder mit KB & Kinder ohne KB \\
\hline$n$ & 132 & 1765 & 99 & 1309 \\
Mädchen in \% & 40,9 & 51,7 & 35,4 & 51,9 \\
Alter in Jahren & 11,9 & 11,3 & 11,8 & 11,3 \\
Anzahl Klassen & 109 & & 77 & \\
\hline
\end{tabular}

Tab. 2 Unterschiede in für ERGMs ein- und ausgeschlossenen Klassen

\begin{tabular}{llll}
\hline & & Eingeschlossene Klassen $(n=77)$ & Ausgeschlossene Klassen $(n=32)$ \\
\hline Klassengröße & Min & 11 & 9 \\
& Max & 25 & 23 \\
& MW & 18,3 & 15,2 \\
Dichte & t-Test & $p<0,001$ & \\
(Freund- & Min & $0,24 / 0,18$ & $0,25 / 0,23$ \\
schaft/ & Max $^{\mathrm{a}}$ & 0,52 & 0,55 \\
Interaktion) & MW $^{\mathrm{a}}$ & 0,35 & 0,39 \\
& t-Test & $p<0,01 / p=0,01$ & \\
\hline
\end{tabular}

${ }^{a}$ Werte für Freundschaft und Interaktion sind gleich

mindestens ein Kind mit SPF aufgrund einer KB unterrichtet. Tab. 1 beschreibt die Stichprobe.

Die für die ERGMs ausgeschlossenen Klassen unterscheiden sich hinsichtlich des Mittelwerts der Anzahl Kinder und der Dichte in den Netzwerken signifikant von den eingeschlossenen Klassen (siehe Tab. 2). Die ausgeschlossenen Klassen sind tendenziell kleiner und dichter. Ein- und ausgeschlossene Klassen überlappen sich aber stark hinsichtlich Größe und Dichte.

\subsection{Datenerhebung}

Das aktive Einverständnis wurde von der Ethikkommission der Fakultät der Universität Bern sowie von den Schulleitungen, Lehrpersonen und den Eltern eingeholt. Die Erhebungen wurden immer von einem Mitglied des Projektteams sowie einem speziell dafür geschulten Masterstudenten, resp. -studentin durchgeführt. Jedes Item wurde vom Untersuchungsleiter laut vorgelesen. Es füllten alle Kinder den Fragebogen gemeinsam im Klassenzimmer aus, wobei das Kind mit einer KB häufig von der Heilpädagogin oder dem Heilpädagogen unterstützt wurde. Einige Kinder mit KB konnten den Fragebogen auch ohne zusätzliche Hilfe ausfüllen.

\subsection{Messinstrumente}

\subsubsection{Soziale Interaktionen im Schulunterricht}

Um die soziale Partizipation zu erfassen, wurde die Peerratingmethode Sozio (Eckhart 2012) verwendet. Sozio baut auf dem Instrument und den Überlegungen von 
Krüger (1976) auf und die enthaltenen Beurteilungskriterien sind sowohl theoretisch wie ethisch geleitet. Positive Interaktionen werden dabei durch wahrgenommene Sprechkontakte angegeben, bei der die Kinder gefragt werden, wie häufig sie mit jeder und jedem ihrer Mitschülerinnen und -schüler in der Schule sprechen (fünfstufige Likertskala von $0=$ fast nie bis $4=$ sehr viel).

Die Berechnung der ERGMs erfolgt mittels dichotomer Angaben (Lusher et al. 2013), weshalb fast nie, selten und manchmal als 0 und viel und sehr viel als 1 codiert werden (Borgatti und Quintane 2018). Durch Aufsummieren der dichotomisierten aus-, resp. eingehenden Sprechkontakte werden individuelle Aktivitäts-, resp. Popularitätswerte generiert.

\subsubsection{Freundschaftsnennungen}

Für die Freundschaftsnetzwerke konnten die Kinder bis zu neun Freundinnen und Freunde aus ihrer Klasse auf leere Linien aufschreiben. Für die deskriptive Statistik wird der Popularitätswert gleich wie beim Sozio hinsichtlich der Klassengröße standardisiert. Für die Beschreibung der Aktivität werden die absoluten Werte verwendet.

\subsubsection{Kontrollvariablen für ERGMs}

Nebst den sozio-demografischen Angaben wie Alter, Geschlecht und SPF können weitere exogene Variablen für die Erklärung der sozialen Netzwerke herangezogen werden. Diese werden nach theoretischen Überlegungen ausgewählt.

Für das schulische Selbstkonzept (SK) wird die leistungsmotivationale Integration aus dem KFDI (Venetz et al. 2014) herangezogen. Die Skala besteht aus vier Items (Bsp.: „Ich kann auch sehr schwierige Aufgaben lösen“) und wird auf einer vierstufigen Likertskala (von $0=$ stimmt nicht bis $3=$ stimmt genau) bewertet (Mittelwert $=2,01$, Cronbach's Alpha=0,78). Es wird davon ausgegangen, dass Kinder, die einen höheren Wert erzielen, lieber in die Schule gehen und deshalb auch mehr Freundschafts- und Interaktionsnennungen machen.

Weiter wurde der sozioökonomische Status (SES) über den Beruf der Eltern erhoben. Der angegebene Beruf wird nach der internationalen Berufsnomenklatur (ISCO-08) codiert und in den ISEI-Wert umgewandelt (Ganzeboom 2010). Für jedes Kind wird nur der höhere ISEI-Wert des jeweiligen Elternteils berücksichtigt. Der SES erreicht eine Spannweite von 14,21 (Putzpersonal) bis 88,96 (Richter/in) mit Mittelwert 51,02. Beim SES wird davon ausgegangen, dass sich ein Homophilieeffekt zeigt: Kinder, die aus ähnlichen sozioökonomischen Verhältnissen stammen, geben eher eine Freundschaftsbeziehung oder Interaktion zueinander an.

Und schließlich wurde als zusätzliche Einflussvariable auf das soziale Netzwerk die Sportvereinszugehörigkeit erhoben $(0=$ nein, $1=$ ja). Es wird angenommen, dass sportliche Aktivität als positives Attribut wahrgenommen wird, weshalb Kinder, die in einem Sportverein sind, mehr Freundschaftsnennungen oder Interaktionen erhalten als Kinder, die nicht in einem Sportverein sind. Von den Kindern mit SPF sind 51 von $99(52 \%)$ in einem Sportverein, bei den Kindern ohne SPF 402 von $1309(31 \%)$. 


\subsection{Datenanalyse}

Die erste Fragestellung wird mittels Intrarater-Reliabilität (Cohen's Kappa $\kappa$, R-package DescTools, Signorell et al. 2021) überprüft. Dabei wird die Übereinstimmung zwischen den dichotomen Angaben im Freundschafts- und Interaktionsnetzwerk berechnet - korrigiert um zufällige Übereinstimmung. Hierfür werden für jedes Kind und jede Klasse die ausgehenden Nennungen und Ratings gepaart. Wenn in einem der beiden Netzwerke ein Wert fehlt, so wird diese Dyade von der Berechnung ausgeschlossen. Für die Beurteilung der $\kappa$-Statistik wird Landis und Koch's (1977) Maßstab angewendet.

Für die zweite Fragestellung werden für jede Klasse für die Schätzung der Freundschafts- und Interaktionsnetzwerke ERGMs gerechnet (R-package ergm, Hunter et al. 2008). In der bisherigen Inklusionsforschung wurde aus den eingehenden Nominationen soziale Akzeptanz- oder Popularitätswerte berechnet, welche dann entweder mittels t-Tests verglichen oder im Rahmen einer Regressionsanalyse verrechnet wurden. Ein anderer Ansatz bilden die ERGMs (Lusher et al. 2013). Hier wird nicht anhand von Netzwerkstatistiken ein individueller Status berechnet, sondern individuelle Attribute der Personen (exogene Faktoren) sowie netzwerkinhärente Strukturen (endogene Faktoren) werden verwendet, um die Beziehungen im sozialen Netzwerk zu erklären (Lusher et al. 2013). In ERGMs wird das Netzwerk als die abhängige Variable betrachtet.

Das Finden der richtigen Modellspezifikation für ein Netzwerk sollte theoriegeleitet geschehen (Lusher et al. 2013). Für unseren Vergleich ist es wichtig, dass pro Klasse jeweils Modelle mit den möglichst gleichen Parametern für das Freundschafts- und Interaktionsnetzwerk geschätzt werden, da die Netzwerkstatistiken von den einbezogenen Parametern abhängen und sonst nicht vergleichbar sind. Deshalb wird folgendermaßen verfahren: Es wird vorgängig ein Vollmodell spezifiziert, das die gewünschten Effekte beinhaltet (siehe Tab. 3).

40 Freundschaftsnetzwerke und 39 Interaktionsnetzwerke konvergierten mit dem Vollmodell. Bei den Netzwerken, die nicht konvergierten, wurde nach einer besseren Modellspezifikation gesucht, indem die Effekte Popularität, Aktivität und Zwei-Pfad schrittweise aus- und eingeschlossen wurden (Reziprozität und Transitivität wurden nie ausgeschlossen, da diese in affektiven oder kollaborativen sozialen Netzwerken wichtige Parameter sind und exogene Variablen wurden nur ausgeschlossen, wenn das Netzwerk nur aufgrund dieser nicht konvergierte). Es wurden nur Klassen in die Analyse eingeschlossen, bei denen beide Netzwerke mit den gleichen Parametern konvergierten (es gab sechs Ausnahmen, bei denen jeweils ein Netzwerk einen Parameter weniger hat als das andere) und bei denen beide Netzwerkanalysen einen guten Daten-Fit aufwiesen. Mit adjustierter Modellspezifikation konnten insgesamt 77 Klassen eingeschlossen werden. Für diese Klassen wurde für jeden Parameter eine univariate random effects Meta-Analyse (R-package metafor, Viechtbauer 2010) gerechnet. Eine Moderator-Analyse gibt an, ob sich der jeweilige Effekt zwischen Freundschafts- und Interaktionsnetzwerk signifikant unterscheidet. 
Tab. 3 Effekte und Effektbeschreibungen für die Spezifikation des Vollmodells

\begin{tabular}{|c|c|c|c|}
\hline & $\begin{array}{l}\text { Modell- } \\
\text { parameter }\end{array}$ & Beschreibung & Visualisierung $^{a}$ \\
\hline \multirow[t]{6}{*}{$\begin{array}{l}\text { Endogene } \\
\text { Faktoren }\end{array}$} & Dichte & $\begin{array}{l}\text { Der Koeffizient gibt die Log-odds aus, für die } \\
\text { Tendenz, dass überhaupt eine Nennung gesendet } \\
\text { wird }\end{array}$ & \\
\hline & Reziprozität & $\begin{array}{l}\text { Die Wahrscheinlichkeit, dass Person } i \text { an Person } j \\
\text { eine Nennung sendet, steigt, wenn bereits eine } \\
\text { Nennung von } j \text { zu } i \text { besteht }\end{array}$ & \\
\hline & Popularität & $\begin{array}{l}\text { Maß für eingehende Nennungen. Ein hoher Wert } \\
\text { sagt aus, dass es im Netzwerk Personen gibt, die } \\
\text { überzufällig häufig Nennungen erhalten }\end{array}$ & \\
\hline & Aktivität & $\begin{array}{l}\text { Maß für ausgehende Nennungen. Ein hoher Wert } \\
\text { sagt aus, dass es im Netzwerk Personen gibt, die } \\
\text { überzufällig häufig Nennungen senden }\end{array}$ & \\
\hline & Transitivität & $\begin{array}{l}\text { Maß für die Struktur, dass Freunde von Freun- } \\
\text { den/Sprechpartner von Sprechpartnern auch eine } \\
\text { Nennung erhalten }\end{array}$ & \\
\hline & Zwei-Pfad & $\begin{array}{l}\text { Der Zwei-Pfad ist eine genestete Struktur in der } \\
\text { Transitivität. Um bei Transitivität nur diesen Effekt } \\
\text { zu schätzen, muss für den Zwei-Pfad kontrolliert } \\
\text { werden }\end{array}$ & \\
\hline \multirow[t]{9}{*}{$\begin{array}{l}\text { Exogene } \\
\text { Faktoren }\end{array}$} & $\begin{array}{l}\text { Geschlechter- } \\
\text { homophilie }\end{array}$ & $\begin{array}{l}\text { Die Wahrscheinlichkeit, dass eine Nennung zwi- } \\
\text { schen zwei gleichgeschlechtlichen Kindern besteht }\end{array}$ & \\
\hline & $\begin{array}{l}\text { Alter (absolu- } \\
\text { te Differenz) }\end{array}$ & $\begin{array}{l}\text { Maß für Alterssimilarität: Ein negatives Vorzei- } \\
\text { chen sagt aus, dass die Wahrscheinlichkeit einer } \\
\text { Nennung steigt, wenn der Altersunterschied klein } \\
\text { ist }\end{array}$ & \\
\hline & $\begin{array}{l}\text { Verein (einge- } \\
\text { hend) }\end{array}$ & $\begin{array}{l}\text { Effekt von Sportvereinszugehörigkeit auf die } \\
\text { Wahrscheinlichkeit, eine Nennung zu erhalten }\end{array}$ & \\
\hline & $\begin{array}{l}\text { Verein (aus- } \\
\text { gehend) }\end{array}$ & $\begin{array}{l}\text { Effekt von Sportvereinszugehörigkeit auf die } \\
\text { Wahrscheinlichkeit, eine Nennung zu senden }\end{array}$ & \\
\hline & $\begin{array}{l}\text { SES (absolute } \\
\text { Differenz) }\end{array}$ & $\begin{array}{l}\text { Mass für die Similarität im sozioökonomischen } \\
\text { Status zweier Kinder: Ein negatives Vorzeichen be- } \\
\text { deutet, dass die Wahrscheinlichkeit einer Nennung } \\
\text { steigt, wenn der Unterschied im SES klein ist }\end{array}$ & \\
\hline & $\begin{array}{l}\text { Schulisches } \\
\text { SK (einge- } \\
\text { hend) }\end{array}$ & $\begin{array}{l}\text { Effekt des schulischen Selbstkonzepts auf die } \\
\text { Wahrscheinlichkeit, eine Nennung zu erhalten }\end{array}$ & \\
\hline & $\begin{array}{l}\text { Schulisches } \\
\text { SK (ausge- } \\
\text { hend) }\end{array}$ & $\begin{array}{l}\text { Effekt des schulischen Selbstkonzepts auf die } \\
\text { Wahrscheinlichkeit, eine Nennung zu senden }\end{array}$ & \\
\hline & $\begin{array}{l}\text { SPF (einge- } \\
\text { hend) }\end{array}$ & $\begin{array}{l}\text { Effekt des SPF auf die Wahrscheinlichkeit, eine } \\
\text { Nennung zu erhalten }\end{array}$ & \\
\hline & $\begin{array}{l}\text { SPF (ausge- } \\
\text { hend) }\end{array}$ & $\begin{array}{l}\text { Effekt des SPF auf die Wahrscheinlichkeit, eine } \\
\text { Nennung zu senden }\end{array}$ & \\
\hline
\end{tabular}

aDie Füllungen haben folgende Bedeutung: Schwarz: Individuum besitzt dieses Attribut (kategorial); Grau: Individuum besitzt dieses Attribut (kardinal); Weiß: Ausprägung des Attributs spielt keine Rolle 


\section{Resultate}

Als erstes wird eine deskriptive Analyse der abhängigen Variablen gezeigt. Bei der Aktivität (ausgehende Nennungen) werden die absoluten Zahlen angegeben (siehe Abb. 1). Die beiden Verteilungen unterscheiden sich, da die Freundschaften auf neun begrenzt sind, während im Sozio die Anzahl ausgehender Nennungen nur durch die Klassengröße beschränkt ist (empirisches Maximum ist 21). Bei den Interaktionen ist der Mittelwert leicht höher als bei den Freundschaftsnennungen $\left(M_{\text {Freund }}=6\right.$, $\left.M_{\text {Inter: }}=6,15, S D_{\text {Freund }}=2,2, S D_{\text {Inter }}=3,45\right)$. Ein Welch t-Test ergibt, dass sich die Mittelwerte der Aktivität nicht signifikant unterscheiden $(t(2393,3)=1,41, p=0,16)$.

Die eingehenden Nennungen sind für beide Instrumente nur durch die Klassengröße beschränkt. Indem die absoluten eingehenden Nennungen durch die Klassengröße geteilt werden, erhält man ein relatives Popularitätsmaß, das über die Klassen hinweg vergleichbar ist. Die Verteilungen dieses Popularitätswerts weisen sehr hohe Überlappungen auf $\left(M_{\text {Freund }}=0,34, M_{\text {Inter }}=0,35, S D_{\text {Freund }}=0,16, S D_{\text {Inter }}=0,15\right.$, siehe Abb. 2). Auch bei der Popularität zeigt ein Welch t-Test, dass sich die Mittelwerte nicht signifikant voneinander unterscheiden $(t(2804,4)=1,31, p=0,19)$.

In Abb. 3 sind die berechneten Kappa-Werte für alle 109 Klassen ersichtlich (ohne Ausreißer: Min=0,4, Max=0,86; Übereinstimmung zwischen moderat und fast perfekt). Wird $\kappa$ für die gesamte Stichprobe mittels Meta-Analyse berechnet, so zeigt sich eine beachtliche Übereinstimmung zwischen Freundschaftsnennungen und dichotomisierten Interaktionsratings $(\kappa=0,64)$. Eine Klasse ist als krasser Ausreißer erkennbar $(\kappa=-0,05)$.

Die inferenzstatistischen Resultate der Netzwerkanalyse sind in Tab. 4 dargestellt. Praktisch alle Netzwerkeffekte weisen die gleichen Vorzeichen und Signifikanzen auf. Unterschiede diesbezüglich gibt es nur in der Aktivität und im schulischen SK (ausgehend) - beide Effekte hängen mit den ausgehenden Nennungen zusammen.

Die Netzwerke können folgendermaßen interpretiert werden: Wenn eine Nennung von $j$ zu $i$ vorliegt, so ist die Wahrscheinlichkeit sehr hoch, dass diese Nennung er-
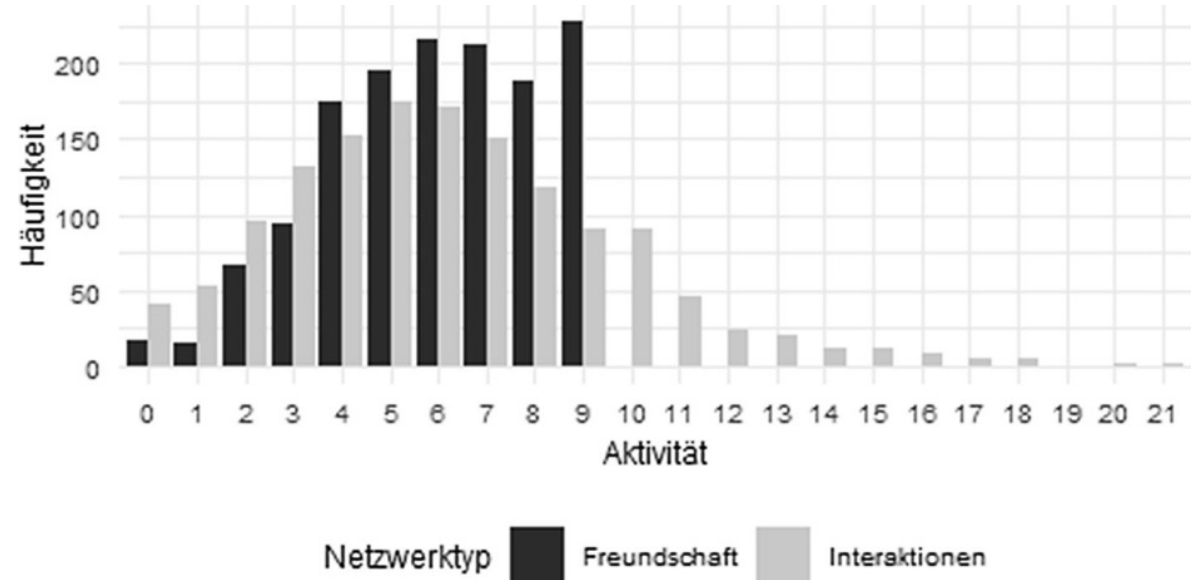

Abb. 1 Verteilungen der Aktivität (ausgehende Nennungen) in absoluten Zahlen 

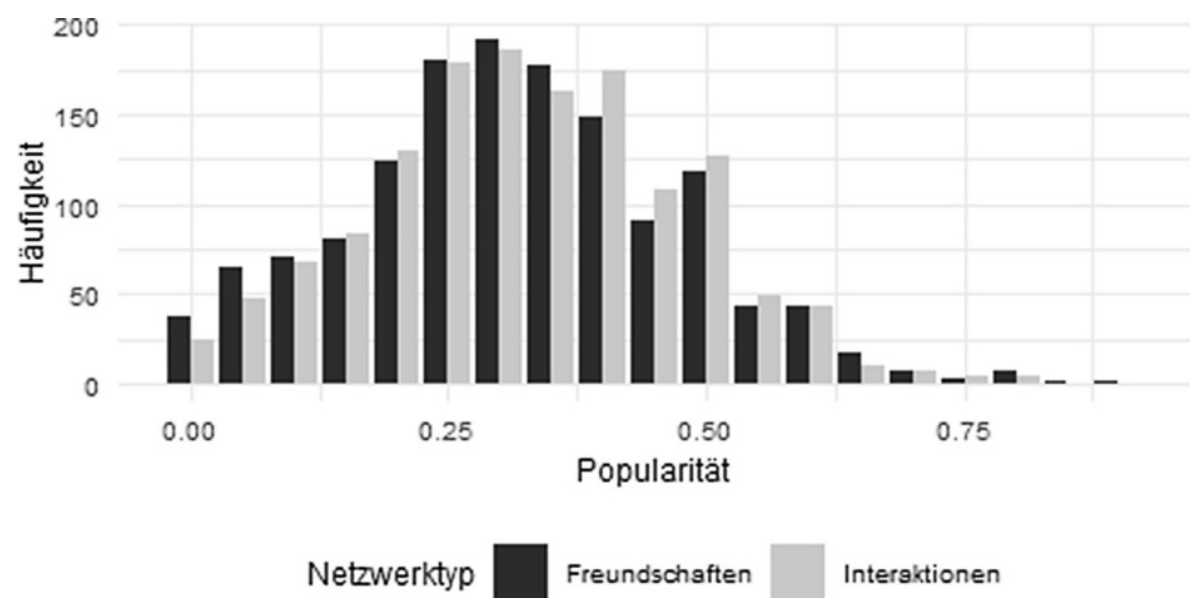

Abb. 2 Verteilungen der eingehenden Nennungen in relativen Zahlen

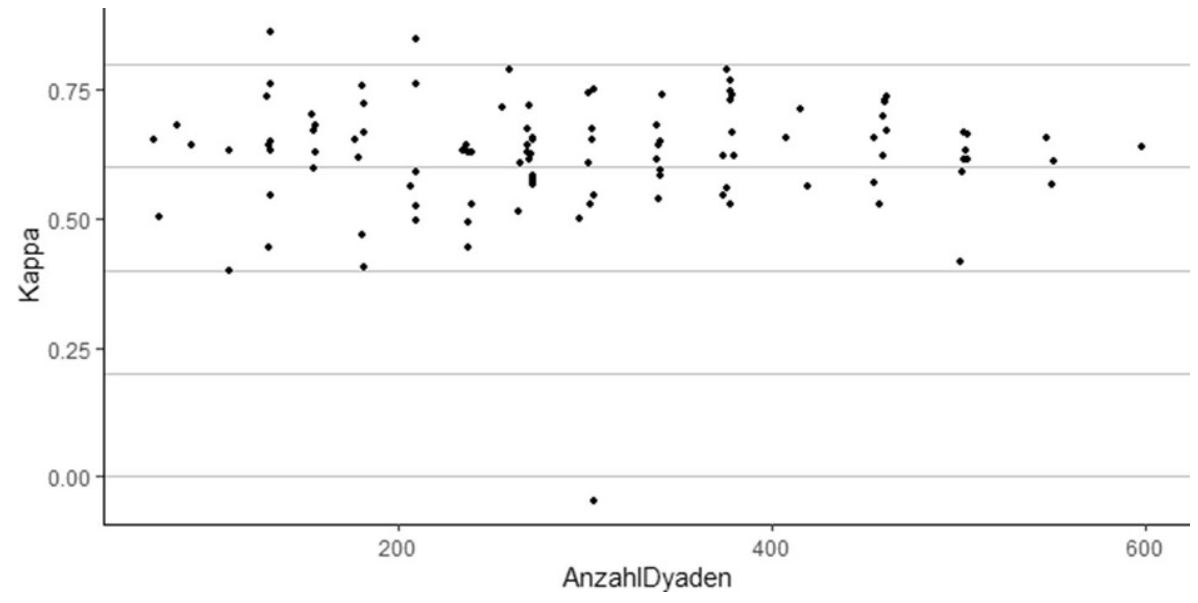

Abb. 3 Kappa-Werte für alle 109 Klassen nach Klassengröße (z. B. bei 20 Kindern in einer Klasse und keinen fehlenden Werten ergeben sich $20 * 20-20=380$ Dyaden)

widert wird (Reziprozität). Auch wird Partnerinnen und Partnern von Partnerinnen und Partnern überzufällig häufig eine Nennung vergeben - d.h. Triaden tendieren dazu, geschlossen zu werden (Transitivität). In den Netzwerken gibt es Kinder, die besonders häufig Nennungen erhalten (Popularität). Im Interaktionsnetzwerk ist zudem ersichtlich, dass es besonders aktive Kinder gibt, jedoch nicht im Freundschaftsnetzwerk (nicht signifikanter Effekt bei Aktivität). Nennungen werden v. a. an gleichgeschlechtliche und gleichaltrige Kinder, sowie an solche mit ähnlichem SES vergeben. Kinder, die in einem Sportverein sind, erhalten weniger Nennungen, wobei sie sich bei den ausgehenden Nennungen nicht von Kindern unterscheiden, die nicht im Verein sind (kein sign. Effekt). Kinder mit höherem schulischem SK erhalten in beiden Netzwerken mehr Nennungen, geben jedoch nur im Interaktionsnetzwerk signifikant mehr Nennungen als Kinder mit niedrigem SK-Wert. Und 
Tab. 4 Random effects Meta-Analysen der ERGMs und Moderatoranalyse

\begin{tabular}{lllll}
\hline Effekt & $\begin{array}{l}\text { Anzahl } \\
\text { Netzwerke }\end{array}$ & Freund & Sprechen & $\begin{array}{l}\text { Moderator- } \\
\text { analyse }\end{array}$ \\
\hline Dichte & 77 & $-3,44(0,16)^{* * *}$ & $-3,95(0,15)^{* * *}$ & 0,017 \\
Reziprozität & 77 & $1,9(0,09)^{* * *}$ & $2,14(0,07)^{* * *}$ & 0,035 \\
Popularität & 53 & $0,16(0,01)^{* * *}$ & $0,13(0,02)^{* * *}$ & 0,244 \\
Aktivität & $65 / 64$ & $0,01(0,02)$ & $0,25(0,01)^{* * *}$ & $<0,0001$ \\
Transitivität & 77 & $0,57(0,07)^{* * *}$ & $0,49(0,06)^{* * *}$ & 0,451 \\
Zwei-Pfad & 67 & $-0,07(0,02)^{* * *}$ & $-0,13(0,01)^{* * *}$ & 0,004 \\
Geschlechterhomophilie & 77 & $1,95(0,1)^{* * *}$ & $1,75(0,09)^{* * *}$ & 0,223 \\
Alter, abs. Differenz & 77 & $-0,24(0,04)^{* * *}$ & $-0,22(0,03)^{* * *}$ & 0,809 \\
Verein (eingehend) & 75 & $-0,19(0,06)^{* *}$ & $-0,13(0,05)^{* *}$ & 0,743 \\
Verein (ausgehend) & 75 & $<0,01(<0,01)$ & $<0,01(<0,01)$ & 0,695 \\
SES, abs. Differenz & 77 & $<-0,01(<0,01)^{+}$ & $<-0,01(<0,01)^{*}$ & 0,991 \\
Schulisches SK (eingehend) & 77 & $0,14(0,04)^{* * *}$ & $0,12(0,04)^{* *}$ & 0,74 \\
Schulisches SK (ausgehend) & 77 & $0,02(0,05)$ & $0,08(0,03)^{* *}$ & 0,22 \\
SPF (eingehend) & 65 & $-0,66(0,12)^{* * *}$ & $-0,61(0,12)^{* * *}$ & 0,756 \\
SPF (ausgehend) & 71 & $-0,11(0,09)$ & $-0,05(0,1)$ & 0,548 \\
\hline
\end{tabular}

In Klammern stehen die Standardfehler

${ }^{* * *} p \leq 0,001 ; * * p \leq 0,01 ;{ }^{*} p \leq 0,05 ;{ }^{+} p \leq 0,1$

schließlich erhalten und senden Kinder mit SPF weniger Nennungen als Kinder ohne SPF, allerdings ist nur der erste Effekt signifikant.

Bei jenen beiden Effekten, die sich nach Erhebungsmethode hinsichtlich Signifikanz unterscheiden, ist der Unterschied bei der Aktivität signifikant (Moderatoranalyse $p<0,0001$ ), jedoch nicht der beim schulischen SK (ausgehend) (Moderatoranalyse $p=0,22$ ).

\section{Diskussion}

\subsection{Netzwerkvergleich}

Die Berechnungen der Intrarater-Reliabilität (Cohen's Kappa) zeigen eine beachtliche Übereinstimmung zwischen den beiden Erhebungsverfahren. Es zeigt sich auch, dass diese stark vom gewählten Grenzwert für die Dichotomisierung der Interaktionen abhängig ist. Wenn man bei der Ausreißerklasse das Rating manchmal statt als 0 als 1 codiert, so springt die Übereinstimmung der dichotomisierten Interaktionen mit den Freundschaftsnennungen von unter 0 auf 0,65 - was einer beachtlichen Übereinstimmung entspricht und zeigt, dass in dieser Klasse die Interaktionen konsistent tiefer eingeschätzt wurden.

Darauf aufbauend zeigen die sozialen Netzwerkanalysen, dass die endogenen und exogenen Faktoren für Freundschaften und Interaktionen praktisch dieselben Ausprägungen haben. Die einzigen Unterschiede finden sich in der Aktivität und im schulischen SK für ausgehende Nennungen, wobei sich die Effekte beim schulischen SK zwischen den Erhebungsmethoden nicht statistisch signifikant unterscheiden. 
Der Unterschied in der Aktivität scheint zudem ein methodisches Artefakt zu sein, da die ausgehenden Nennungen für Freundschaften auf neun begrenzt waren, während sie bei Sozio nur durch die Klassengröße limitiert sind. Aufgrund statistischer Auswertungsbeschränkungen (Cillessen und Marks 2017) waren früher limitierte Nominationsverfahren vertretbar. Mithilfe der modernen Netzwerkanalyse können allerdings auch unlimitierte Angaben statistisch ausgewertet werden, weshalb limitierte Verfahren obsolet sind. Aus pragmatischen Gründen wurde in unserer Studie für neun Nennungen optiert: Erstens ist das Freundinnen- und Freunde-Aufschreiben zeitintensiv und zweitens sollte ein wahlloses Nominieren verhindert werden für die statistischen Auswertungen wird jedoch von diesem Vorgehen abgeraten.

Es muss beachtet werden, dass ERGMs allein keine Validierung der Messmethoden zulassen. In ERGMs wird gemessen, ob sich die Verteilung der in der Analyse einbezogenen Strukturen überzufällig stark von jenen in Zufallsnetzwerken unterscheidet. Dies ist in Freundes- und Interaktionsnetzwerken in sehr ähnlichem Ausmaß der Fall. Die Validierung der Übereinstimmung erfolgt durch die IntraraterReliabilität. Folglich sind die ERGMs als inhaltliche Ergänzung zu sehen, die zeigt, dass sich die sozialen Netzwerke unabhängig der Erhebungsmethode sehr ähnlich gestalten.

Anhand der Intrarater-Reliabilität und der ERGMs kommen wir zum Schluss, dass sich die Freundschaftsnetzwerke nicht bedeutsam von den Interaktionsnetzwerken unterscheiden, weswegen soziale Partizipation generell mit dem Sozio (Eckhart 2012) erhoben werden kann und - in der Inklusionsforschung - sollte. Das mögliche Argument, dass sich Freundschaftswahlen qualitativ von Interaktionsratings unterscheiden, da es bei Freundschaften in erster Linie um den emotional-affektiven Aspekt einer Beziehung geht und bei wahrgenommenen Interaktionen dieser Aspekt fehlt, wird dadurch irrelevant, dass es für die abhängige Variable - also die sozialen Netzwerke - keine Rolle spielt, ob sie mittels Freundschaften oder Interaktionen erfasst werden.

\subsection{Anwendungsbereich}

Soziometrische Untersuchungen werden durchgeführt, um daraus - falls nötig Interventionen für die bessere Integration bestimmter Kinder vorzunehmen. In gängigen Peernominationen werden auch negative Kriterien erfragt, damit die Kinder einem sozialen Status nach Coie et al. (1982) zugeordnet werden können. Der Nutzen liegt darin, dass Interventionen auf den Statustyp angepasst werden können (z.B. für Abgelehnte vs. Vernachlässigte). Jedoch wird eine Fokussierung auf den sozialen Status aus ethischer Sicht kritisiert (Child und Nind 2012). Zudem können Interventionen auch aus der hier vorgestellten Analyse mittels ERGMs abgeleitet werden. Eine solche Intervention würde auf das ganze Netzwerk abzielen, was mit den Schlussfolgerungen von Garrote et al. (2017) zu Interventionen in inklusiven Klassen konform ist, wonach Interventionen v. a. dann erfolgreich sind, wenn sie die ganze Gruppe einbeziehen. Eine netzwerkgeleitete Intervention sollte die vorhandenen Strukturen im Netzwerk ausnutzen, um sozial weniger eingebundene Kinder zu mehr Interaktionen zu verhelfen. Die sozialen Netzwerke scheinen stark durch Reziprozität, Transitivität, Aktivität und Geschlechterhomophilie geprägt zu sein. 
Deshalb kann vorgeschlagen werden, Kinder, die aufgrund exogener Faktoren signifikant weniger Interaktionen erhalten, bei Arbeiten in geschlechtshomogene Gruppen mit besonders aktiven Kindern zusammenzutun. Durch die Prozesse der Reziprozität und Transitivität könnten dann die betroffenen Kinder profitieren.

\subsection{Limitierungen und Ausblick}

Die Verwendung der neusten Netzwerkanalyse ist eindeutig eine Stärke dieses Artikels. Trotzdem ergeben sich gerade auch im methodisch-analytischen Bereich Limitierungen. Aus technischen Gründen war es nicht möglich, die Interaktionen auch als gewichtete Netzwerke zu rechnen. Die Dichotomisierung geht mit einem Informationsverlust einher, der hier in Kauf genommen werden musste. Das Rating per se ist eigentlich ein weiteres Argument für ein Ratingverfahren gegenüber einer rein dichotomen Nomination, da die Beziehung zwischen Subjekten differenzierter erfasst wird. Eine weitere Meta-Analyse mit gewichteten Netzwerken würde die empirische Grundlage weiter bereichern. Weiter wären längsschnittliche Daten wünschenswert. Mittels stochastischen Akteur-orientierten Modellen (Snijders et al. 2010) können in längsschnittlichen Netzwerkanalysen Selektions- und Einflussmechanismen unterschieden werden, was in ERGMs nicht möglich ist.

Schließlich muss erwähnt werden, dass von 109 Klassen 32 (29\%) für ERGMs ausgeschlossen wurden, da die Modelle nicht konvergierten. Die Nicht-Konvergenz kann verschiedene Gründe haben, einer ist die Kollinearität von Variablen (z.B. Reziprozität und Geschlechterhomophilie). Ein anderer möglicher Grund ist, dass die spezifizierten Modelle für diese Klassen nicht passen - d. h., es hätten andere Effekte berücksichtigt werden müssen. Evtl. hätten sich in den ausgeschlossenen Klassen Unterschiede in den Freundschafts- und Interaktionsnetzwerken gezeigt. Gleichwohl darf auch darauf hingewiesen werden, dass die Meta-Analyse auf 77 Klassen beruht und dies eine starke Evidenz für die vorgestellten Resultate bedeutet.

\subsection{Konklusion}

Die Diskussion um ethisch vertretbare Instrumente in der Forschung kann nie als abgeschlossen gelten (APA 2017). Es gibt bereits Bemühungen, die ethische Vertretbarkeit durch empirische Untersuchungen zu stützen. Aufgrund methodischer Schwierigkeiten sehen wir bei diesem Ansatz nur geringe Erfolgschancen. Stattdessen schlagen wir vor, auf die Berücksichtigung ethischer Aspekte bei der Testkonstruktion Wert zu legen. Unsere Untersuchung hat gezeigt, dass die gängige Freundschaftswahl verglichen mit dem hier verwendeten Interaktionsrating eine beachtliche Übereinstimmung sowie starke inhaltliche Ähnlichkeit aufweisen. Gerade in der Inklusionsforschung ist es wichtig, dass Forscherinnen und Forscher für den ethischen Aspekt soziometrischer Instrumente sensibel sind.

Funding Open access funding provided by University of Bern

Open Access Dieser Artikel wird unter der Creative Commons Namensnennung 4.0 International Lizenz veröffentlicht, welche die Nutzung, Vervielfältigung, Bearbeitung, Verbreitung und Wiedergabe in 
jeglichem Medium und Format erlaubt, sofern Sie den/die ursprünglichen Autor(en) und die Quelle ordnungsgemäß nennen, einen Link zur Creative Commons Lizenz beifügen und angeben, ob Änderungen vorgenommen wurden.

Die in diesem Artikel enthaltenen Bilder und sonstiges Drittmaterial unterliegen ebenfalls der genannten Creative Commons Lizenz, sofern sich aus der Abbildungslegende nichts anderes ergibt. Sofern das betreffende Material nicht unter der genannten Creative Commons Lizenz steht und die betreffende Handlung nicht nach gesetzlichen Vorschriften erlaubt ist, ist für die oben aufgeführten Weiterverwendungen des Materials die Einwilligung des jeweiligen Rechteinhabers einzuholen.

Weitere Details zur Lizenz entnehmen Sie bitte der Lizenzinformation auf http://creativecommons.org/ licenses/by/4.0/deed.de.

\section{Literatur}

Americal Psychological Association (2017). Ethical principles of psychologists and code of conduct. https://www.apa.org/ethics/code/ethics-code-2017.pdf. Zugegriffen: 1. Nov. 2020.

Bell-Dolan, D., \& Wessler, A. E. (1994). Ethical administration of sociometric measures: procedures in use and suggestions for improvement. Professional Psychology: Research and Practice, 25(1), 23-32.

Borgatti, S.P., \& Quintane, E. (2018). Techniques: dichotomizing a network. Connections, 38(1), 1-11.

Child, S., \& Nind, M. (2012). Sociometric mehtods and difference: a force for good-or yet more harm. Disability \& Society. https://doi.org/10.1080/09687599.2012.741517.

Cillessen, A.H.N., \& Marks, P.E.L. (2011). Conceptualizing and measuring popularity. In A.H. N. Cillessen, D. Schwartz \& L. Mayeux (Hrsg.), Popularity in the peer system (S. 25-56). New York: Guilford.

Cillessen, A.H.N., \& Marks, P.E.L. (2017). Methodological choices in peer nomination research. In P.E. L. Marks \& A.H.N. Cillessen (Hrsg.), New directions in peer nomination methodology. New directions for child and adolescent development, (Bd. 157, S. 21-44). Online Library: Wiley.

Coie, J.D., Dodge, K. A., \& Coppotelli, H. (1982). Dimensions and types of social status: a cross-age perspective. Developmental Psychology, 18(4), 557-570.

Eckhart, M. (2005). Anerkennung und Ablehnung in Schulklassen. Einstellungen und Beziehungen von Schweizer Kindern und Immigrantenkindern. Bern: Haupt.

Eckhart, M. (2012). Soziale Integrationsprozesse in Schulklassen: Methodische Annäherungen und Entwicklung eines Computerprogramms zur Analyse sozialer Prozesse in Schulklassen (Sozio). In A. Lanfranchi \& J. Steppacher (Hrsg.), Schulische Integration gelingt: Gute Praxis wahrnehmen, Neues entwickeln (S. 136-147). Bad Heilbrunn: Klinkhardt.

Ganzeboom, H. B. G. (2010). A new international socio-economic index [ISEI] of occupational status for the international standard classification of occupation 2008 [ISCO-08] constructed with data from the ISSP 2002-2007; With an analysis of quality of occupational measurement in ISSP. https://www. researchgate.net/publication/242660186. Zugegriffen: 1. Nov. 2020. Conference Paper.

Garrote, A. (2016). Soziale Teilhabe von Kindern in inklusiven Klassen. Empirische Pädagogik, 30(1), 67-80.

Garrote, A., Sermier Dessemontet, R., \& Moser Opitz, E. (2017). Facilitating the social participation of pupils with special educational needs in mainstream schools: a review of school-based interventions. Educational Research Review, 20, 12-23.

Hayvren, M., \& Hymel, S. (1984). Ethical issues in sociometric testing: Impact of sociometric measures on interaction behavior. Developmental Psychology, 20(5), 844-849.

Henke, T., Bogda, K., Lambrecht, J., Bosse, S., Koch, H., Maaz, K., \& Spörer, N. (2017). Will you be my friend? A multilevel network analysis of friendships of students with and without special educational needs backgrounds in inclusive classrooms. Zeitschrift für Erziehungswissenschaft, 20, 449-474.

Hunter, D.R., Handcock, M.S., Butts, C. T., Goodreau, S. M., \& Morris, M. (2008). ergm: a package to fit, simulate and diagnose exponential-family models for networks. Journal of Statistical Software, 24(3), nihpa54860. https://doi.org/10.18637/jss.v024.i03.

Iverson, A.M., \& Iverson, G.L. (1996). Children's long-term reactions to participating in sociometric assessment. Psychology in the Schools, 33, 103-112. 
Koster, M., Nakken, H., Pijl, S. J., \& van Houten, E. (2009). Being part of the peer group: a literature study focusing on the social dimension of inclusion in education. International Journal of Inclusive Education, 13(2), 117-140.

Krüger, H.-P. (1976). Soziometrie in der Schule. Verfahren und Ergebnisse zu sozialen Determinanten der Schülerpersönlichkeit. Weinheim: Beltz.

Landis, R. J., \& Koch, G. G. (1977). The measurement of observer agreement for categorical data. Biometrics, 33(1), 159-174.

Lusher, D., Koskinen, J., \& Robins, G. (2013). Exponential random graph models for social networks: theory, methods, and applications. Cambridge: Cambridge University Press.

Mayeux, L., Underwood, M.K., \& Risser, S.D. (2007). Perspectives on the ethics of sociometric research with children. Merril-Palmer Quarterly, 53(1), 53-78.

Moreno, J.L. (1996). Die Grundlagen der Soziometrie. Wege zur Neuordnung der Gesellschaft. Wiesbaden: Springer. Unveränderter Nachdruck der 3. Auflage 1974

Peery, J.C. (1979). Popular, amiable, isolated, rejected: a reconceptualization of sociometric status in preschool children. Child Development, 50, 1231-1234.

Schweizerische Konferenz der kantonalen Erziehungsdirektoren (2014). Standardisiertes Abklärungsverfahren. Instrument des Sonderpädagogik-Konkordats als Entscheidungsgrundlage für die Anordnung verstärkter individueller Massnahmen - Handreichung. Bern: Ediprim.

Signorell, A., et al. (2021). DescTools: tools for descriptive statistics. R package version 0.99.42. https:// cran.r-project.org/package=DescTools. Zugegriffen: 10. Juli 2021

Snijders, T. A. B., van de Bunt, G.G., \& Steglich, C.E.G. (2010). Introduction to stochastic actor-based models for network dynamics. Social Networks, 32, 44-60.

Underwood, M. K., Mayeux, L., Risser, S.D., \& Harper, B. (2006). The ecstasy and the agony of collecting sociometric data in public school classrooms: challenges, community concerns, and pragmatic solutions. In B. Leadbeater, E. Banister, C. Benoit, M. Jansson, A. Marshall \& T. Riecken (Hrsg.), Ethical issues in community-based research with children and youth (S. 93-110). Toronto: University of Toronto Press.

Venetz, M., Zurbriggen, C., \& Eckhart, M. (2014). Entwicklung und erste Validierung einer Kurzversion des „Fragebogens zur Erfassung von Dimensionen der Integration von Schülern (FDI 4-6)“ von Haeberlin, Moser, Bless und Klaghofer. Empirische Sonderpädagogik, 2, 99-113.

Viechtbauer, W. (2010). Conducting meta-analyses in R with the metafor package. Journal of Statistical Software. https://doi.org/10.18637/jss.v036.i03. 